\begin{tabular}{|c|c|}
\hline Citation & $\begin{array}{l}\text { K.M. Schultz, L. Campo-Deano, A.D. Baldwin, K.L. Kiick, C. Clasen, E.M. } \\
\text { Furst, } \\
\text { Electrospinning covalently cross-linking biocompatible hydrogelators } \\
\text { Polymer } 54(1)(2013) 363-371 .\end{array}$ \\
\hline Archived version & $\begin{array}{l}\text { Author manuscript: the content is identical to the content of the published } \\
\text { paper, but without the final typesetting by the publisher }\end{array}$ \\
\hline Published version & $\begin{array}{l}\text { The final publication is available via } \\
\text { http://dx.doi.org/10.1016/j.polymer.2012.09.060 }\end{array}$ \\
\hline Journal homepage & http://www.sciencedirect.com/science/journal/00323861 \\
\hline Author contact & your email christian.clasen@cit.kuleuven.be \\
\hline IR & url in Lirias https://lirias.kuleuven.be/handle/123456789/357129 \\
\hline
\end{tabular}

(article begins on next page) 


\title{
Electrospinning covalently cross-linking biocompatible hydrogelators
}

\author{
Kelly M. Schultz ${ }^{\mathrm{a}}$, Laura Campo-Deaño ${ }^{\mathrm{b}}$, Aaron D. Baldwin ${ }^{\mathrm{c}}$, Kristi L. \\ Kiick $^{\mathrm{c}}$, Christian Clasen ${ }^{\mathrm{b}}$, Eric M. Fursta,* \\ ${ }^{a}$ Department of Chemical and Biomolecular Engineering and Center for Molecular and \\ Engineering Thermodynamics, University of Delaware, 150 Academy St., Newark, DE \\ 19716, USA \\ ${ }^{b}$ Department of Chemical Engineering, Katholieke Universiteit Leuven, Willem de \\ Croylaan 46, B-3001 Leuven, Belgium \\ ${ }^{c}$ Department of Materials Science and Engineering, University of Delaware, 201 DuPont \\ Hall, Newark, DE 19716, USA
}

\begin{abstract}
Many hydrogel materials of interest are homogeneous on the micrometer scale. Electrospinning, the formation of sub-micrometer to micrometer diameter fibers by a jet of fluid formed under an electric field, is one process being explored to create rich microstructures. However, electrospinning a hydrogel system as it reacts requires an understanding of the gelation kinetics and corresponding rheology near the liquid-solid transition. In this study, we correlate the structure of electrospun fibers of a covalently crosslinked hydrogelator with the corresponding gelation transition and kinetics. Polyethylene oxide (PEO) is used as a carrier polymer in a chemically crosslinking poly(ethylene glycol)-high molecular weight heparin (PEG-HMWH) hydrogel. Using measurements of gelation kinetics from multiple particle tracking microrheology (MPT), we correlate the material rheology with the the formation of stable fibers. An equilibrated, cross-linked hydrogel is then spun and the PEO is dissolved. In both cases, microstructural features of the electrospun fibers are retained, confirming the covalent nature of the network. The ability to spin fibers of a cross-linking hydrogel system ultimately enables the engineering of materials and microstructural length scales
\end{abstract}

*Department of Chemical and Biomolecular Engineering and Center for Molecular and Engineering Thermodynamics, University of Delaware, 150 Academy St., Newark, DE 19716, USA, furst@udel.edu 
suitable for biological applications.

Keywords: microrheology, electrospinning, hydrogels

\section{Introduction}

Synthetic scaffolds are designed with increasing complexity through the use of chemical and physical cues with the goal of controlling and manipulating cellular processes, such as motility and proliferation. One method of creating extracellular matrix (ECM) mimics is by processing polymeric materials using electrospinning. This creates continuous fibers that range in diameter from nano- to micrometers $[1,2,3,4,5,6,7]$. Due to the unique properties of these fibers, such as high porosity and surface area of spun mats, their application areas include tissue engineering, drug delivery, composites, and electrical and optical materials $[8,9,10,2,11,12,13,14,15,16,17,18$, $19,20,21,22,23,24,25,26,27,28,29,30,31,32,33,34,35,6,7,36]$. The characterization of the material, prior to electrospinning, enables the design of the final properties of the material scaffold and also conserves time and expensive synthetic materials used to achieve a continuous fiber. To meet this end, our work uses multiple particle tracking microrheology (MPT) to identify material properties of a biocompatible hydrogelator that will enable continuous fiber formation using electrospinning. Fibers of this cross-linked gel will create scaffolds that can be used in therapeutic and biological applications, such as wound healing and tissue regeneration.

Three techniques are predominately used to create nanofibrous cell scaffolds: self-assembly, phase separation and electrospinning. Self-assembled fibers have length scales that are at the lower limit of the ECM, while phase separation only occurs in a select number of polymers. Therefore, electrospinning is an essential and versatile technique to create scaffolds $[8,17,18,37,38]$. Furthermore, methods for orienting fibers enables the engineering of specific biological microenvironments. Functionalization of these scaffolds with bioactive species is done through encapsulation or attachment, facilitating cell-material communication and interactions $[10,11,12,13,14$, $15,16,17,18,19,20,21,22,23,24,25,26,27,28,29,30,31,32,33,34,35]$. Many studies have documented the use of electrospun fibers for biological applications, including their use as scaffolds for supporting nerve regeneration and creating fibers of composite materials such as polyethylene oxide (PEO) and DNA [39, 40, 12, 14, 20]. Advantages of electrospinning polymers for 
use in biological applications include high surface-to-volume ratio, tunable porosity, the ability of the fiber mat to conform to many shapes and sizes and the scaffold properties and functionality can be controlled easily by varying material properties prior to spinning [9].

In electrospinning, electrostatic forces supplement or replace mechanical forces to create fibers that are much thinner than those achieved by using conventional mechanical extrusion $[1,2,3,4,5,6,7]$. A polymeric solution is supplied through a charged capillary above a grounded electrode. The electric potential created through the applied voltage between the capillary and ground, on the order of kilovolts, is the driving force to create a Taylor cone at the tip of the capillary. The Taylor cone ejects a jet of charged solution that thins under the development of bending instabilities in order to maximize the distance between the charges on the jet. The loss of solvent during the jet thinning ultimately leads to the formation of solid polymer fibers $[1,2,3,4,5,6,7]$. A delicate balance of the polymeric solution flow rate, applied voltage and separation of the ground and capillary results in jet and fiber dimensions on the order of nano- to micrometers $[2,3,36]$.

The literature provides few instances of electrospinning hydrogelators. These materials are difficult to spin due to their high moduli, which frequently leads to extrusion from the capillary. This is a disadvantage in using this process to create material scaffolds. Cross-linked electrospun fibers have been made using two techniques: first, engineering the reaction time scale such that the material gels during (or shortly after) spinning, and second, by cross-linking the nanofibers after they are spun [41, 42]. Using photo-induced polymerization the molecules within the fibers can also be cross-linked during the electrospinning process $[43,44]$.

The present work focuses on processing poly(ethylene glycol)-high molecular weight heparin (PEG-HMWH) hydrogels using electrospinning. The aim is to demonstrate the utility of empirical hydrogelation state diagrams, described in previous work $[45,46,47]$, for predicting the electrospinnability and to create mats of these hydrogel materials that can be used in wound healing and tissue regeneration applications [48]. These gelation state diagrams enable facile determination of the appropriate hydrogel composition that will electrospin, specifically a cross-linked gel with a low cross-link density. The state information eliminates trial and error experiments. 


\section{Experimental}

\subsection{Materials}

The hydrogel is composed of a multi-functional high molecular weight heparin backbone (HMWH, $M_{n}=15000 \mathrm{~g} / \mathrm{mol}, 75 e^{-}$, Celsius) and a poly(ethylene glycol) cross-linker functionalized with thiol end groups (PEG, $M_{n}=2000$ $\mathrm{g} / \mathrm{mol}$ and $5000 \mathrm{~g} / \mathrm{mol}$, Sigma Aldrich). The HMWH backbone is functionalized with maleimide-NHS groups with the number of functional groups determined by ${ }^{1} \mathrm{H}$ NMR spectroscopy. These maleimide groups react with the thiol end groups on the linear PEG cross-linker to form a chemically cross-linked hydrogel. Details of the synthesis of this material are described elsewhere [49]. The hydrogel components are dissolved separately in phosphate buffered saline $(1 \times$ PBS, pH 7.4), prepared from a standard protocol. To increase the viscosity, polyethylene oxide (PEO, $M_{n}=1000000 \mathrm{~g} / \mathrm{mol}$, Union Carbide) is used as a carrier polymer. A solution of $3 \mathrm{wt} \%$ is prepared in distilled water by gently stirring in the polymer to speed dissolution. Additionally, we perform control experiments with unfunctionalized HMWH and PEG precursor solutions.

\subsection{Methods}

\subsubsection{Multiple particle tracking microrheology}

Previous to electrospinning, hydrogel material properties are characterized using multiple particle tracking microrheology (MPT). Fluorescently labeled colloidal probe particles $(2 a=1.04 \pm 0.02 \mu \mathrm{m}$, where $a$ is the particle radius, Polysciences, Inc.) are dispersed into the HMWH precursor solution. The Brownian movement of the probe particles is used to extract rheological properties of the solutions and mixtures. The hydrogelation reaction is initiated by mixing of the HMWH and PEG solutions. For the hydrogelation reaction, data are collected every 2.5 minutes after the initiation of the reaction and until 30 minutes after particles are completely arrested in the gel matrix [46]. A high-throughput screening technique is used to collect data for state diagrams of the hydrogels equilibrated for at least 2 hours $[45,46]$. Details of these methods are published previously [45, 46, 47].

Briefly, fluorescent video microscopy is used to capture the Brownian motion of the probe particles. The movement of the particles is magnified $63 \times$ (water immersion objective, N. A. 1.2, 1× optovar, Carl Zeiss, Inc.) and data are collected on an inverted microscope (Axiovert 200, Carl Zeiss, Inc.) using a CMOS high-speed camera (Phantom v5.1, 1024×1024 pixels, 
Vision Research Inc.). Videos are captured at 30 frames/s and an exposure time of $1 \mathrm{~ms}$, to minimize static and dynamic particle tracking errors [50]. Approximately 100 particles are tracked in each 800 frame movie yielding sufficient statics to determine material rheological properties $[51,52]$. The brightness-weighted centroid of each particle is determined in each frame and these positions are linked together into trajectories using particle tracking algorithms [53]. From these trajectories, the ensemble-averaged mean-squared displacement (MSD), $\left\langle\Delta r^{2}(\tau)\right\rangle$, is calculated.

The MSD is related to the material properties using the Generalized Stokes-Einstein relation (GSER)

$$
\left\langle\Delta r^{2}(\tau)\right\rangle=\frac{k_{B} T}{\pi a} J(\tau),
$$

where $k_{B} T$ is the thermal energy, $a$ is the probe particle radius and $J(\tau)$ is the material creep compliance. The creep compliance is defined as

$$
\gamma(t)=\int_{0}^{t} J\left(t-t^{\prime}\right) \sigma\left(t^{\prime}\right) d t^{\prime}
$$

where $\gamma(t)$ is the sample strain that evolves from the applied stress $\sigma(t)$ $[54,55]$. These measurements characterize the cross-linked material before addition of a carrier polymer, but give reliable information about the state of the material and the relative strength of that gel. Measurements are taken both after the material has equilibrated and during the hydrogelation reaction and serve as a guide to determine whether a hydrogel can be electrospun. Measurements of the uncross-linked material include the carrier polymer.

\subsubsection{Sample preparation}

Cross-linking hydrogel electrospinning experiments are performed with solutions that are equilibrated for approximately 15 mins and also with a solution that is evolving through the gelation reaction. The procedure for spinning these solutions is similar. Before electrospinning is begun, the crosslinker and backbone solutions are weighed out and dissolved in $1 \times$ PBS separately. Hydrogels are allowed to equilibrate for 15 minutes prior to spinning, this equilibration time is chosen from microrheological experiments of the gelation kinetics showing no change in the rheological properties after approximately 5 mins. For experiments during gelation, the HMWH backbone and PEG cross-linker solutions are mixed prior to spinning, initiating the 
Table 1: Solution compositions for electrospinning experiments.

\begin{tabular}{ccccc}
\hline $\begin{array}{c}\text { HMWH } \\
\begin{array}{c}\text { concentration } \\
(\text { wt } \%)\end{array}\end{array}$ & $\begin{array}{c}\text { HMWH } \\
\text { functionality }\end{array}$ & $\begin{array}{c}\text { PEG } \\
\text { concentration } \\
\text { (wt } \%)\end{array}$ & $\begin{array}{c}\text { PEG } \\
\text { functionality }\end{array}$ & $\begin{array}{c}\text { PEO } \\
\text { concentration } \\
\text { (wt } \%)\end{array}$ \\
\hline 0 & 0 & 0 & 0 & 1 \\
0 & 0 & 0 & 0 & 3 \\
0 & 0 & 1 & 0 & 1 \\
0 & 0 & 1 & 0 & 2 \\
1 & 0 & 0 & 0 & 1 \\
1 & 0 & 0 & 0 & 2 \\
1 & 0 & 1 & 0 & 1 \\
1 & 0 & 1 & 0 & 2 \\
4 & 3.9 & 1 & 2 & 1 \\
3 & 3.9 & 2 & 2 & 0 \\
1 & 6.3 & 1 & 2 & 2 \\
1 & 6.3 & 1 & 2 & 1 \\
\hline
\end{tabular}

gelation reaction. The carrier polymer solution, PEO, is then added to the hydrogel solution to obtain the appropriate final composition. The hydrogel material is loaded into the syringe, which will contact the upper plate of the electrospinning apparatus, as described below. Control experiments are conducted using solutions of PEO, unfunctionalized PEG and unfunctionalized HMWH. All solutions are unreactive polymer mixtures with no cross-linking. The sample solutions are mixed thoroughly outside of the electrospinning equipment using a vortex mixer.

The compositions of the materials used in all experiments are summarized in Table 1.

\subsubsection{Electrospinning experimental apparatus}

A parallel plate electrospinning apparatus is used in these investigations. The apparatus is detailed in the supplemental information.

The electrospinning apparatus has three parameters that can be varied: flow rate, separation of plates and applied voltage. These parameters are optimized by spinning a $3 \mathrm{wt} \%$ PEO solution. This solution is well studied 
in the literature and parameters for spinning in a point-plate apparatus are outlined in work published by Helgeson et al. [2, 56]. Due to the different electrospinning apparatus, optimization begins with the parameters outlined in the literature and are adjusted for the equipment. To spin the PEO solution, three plate separations are used: 14.5, 15.5 and $16.7 \mathrm{~cm}$. Placing the plates too close results in electrospraying because the the electric field produced is too strong [57]. The upper separation limit is the maximum plate separation achievable with this electrospinning equipment, $16.7 \mathrm{~cm}$. A flow rate between $500-750 \mu \mathrm{L} / \mathrm{hr}$ is established by empirical methods. Volumetric flow rates outside of this interval resulted in the polymer solution drying on the needle at the lower limit and at the upper limit droplets are formed too quickly for the fluid to reach the critical charge density to form a Taylor cone. The final parameter optimized is the applied voltage. In the parallel-plate apparatus, the voltages are approximately $4 \times$ larger than those reported by Helgeson et al. [2]. Through experimentation, fibers form at voltages from $20 \mathrm{kV}$ to the maximum voltage achievable by the equipment, $25 \mathrm{kV}$. Using these ranges of operating parameters, all experiments keep a constant flow rate of $750 \mu \mathrm{L} / \mathrm{hr}$ and an applied voltage of $25 \mathrm{kV}$.

\subsubsection{Dissolution of carrier polymer}

The PEO carrier polymer is subsequently dissolved from all electrospun fibers. Dissolving the PEO ensures that the remaining microstructure is unique to the cross-linked hydrogel fibers. The procedure outlined by Ji et al. is followed [41]. A piece of the electrospun mat is cut out and fixed to a SEM stub (Aluminum SEM Specimen Mount, Electron Microscopy Sciences) with carbon tape. All stubs are placed in a petri dish with a water saturated atmosphere, to prevent evaporation. Evaporation of the water would cause the fibers to collapse making the analysis of microstructure impossible. The fibers are then immersed in water and covered, taking care that the water stays on the stub and does not run off and remove the fibers. The fibers soak in water for 24 hours. The SEM stubs are then removed from the water rich environment and lyophilized for 24 hours. After this treatment, the fibers are imaged using SEM and the remaining microstructure is observed.

\subsubsection{Scanning electron microscopy}

Hydrogel fibers before and after dissolution are imaged using a field emission scanning electron microscope (JEOL Ltd., JSM-7400F, Japan). Fibers spun onto aluminum foil are fixed directly to a SEM stub using carbon tape 
and are sputtered with gold for $45 \mathrm{~s}$. Fiber and pore diameters are calculated using image analysis in ImageJ (National Institutes of Health, Bethesda, Maryland). Average feature dimensions are measured using at least one hundred separate features for each sample condition.

\section{Results}

Within the course of this work, many cross-linking hydrogel compositions and unreactive samples were spun to validate that chemical cross-links in the hydrogel were responsible for the unique microstructures. In the following results and discussion section, only key experiments are discussed. All other sample conditions used in this study are summarized in Table 1 and are reported in the supplemental material.

\subsection{Control experiments}

Control experiments are performed to verify that the electrospun crosslinking hydrogel material results in a unique porous microstructure that cannot be achieved by spinning a mixture of the same uncross-linked polymers. The concentrations are chosen to be identical to the equilibrated hydrogel composition selected from the gelation state diagram.

\subsubsection{Polyethylene oxide}

A $3 \mathrm{wt} \%$ PEO solution is used to optimize the electrospinning parameters and verify that the PEO does not yield a fiber microstructure similar to that the cross-linked electrospun hydrogel. The polymer concentration is the same as the total polymer concentration as the hydrogel mixtures. A 1 wt\% PEO solution (supplemental information) was also spun to mimic the actual PEO concentration within several of the hydrogel samples.

The $3 \mathrm{wt} \%$ PEO solution spins intermittently at 14.5 and $15.5 \mathrm{~cm}$ plate separations, but continuous spinning occurs for 3 hours at the $16.7 \mathrm{~cm}$ separation. The resulting fibers exhibit elongated beads, but the number of beads diminishes as the separation distance increases. Applying the gentle dissolution procedure described above, most of the PEO dissolves. For the 14.5 and $16.7 \mathrm{~cm}$ separations, a structure with very thin fibers remains. At $15.5 \mathrm{~cm}$, a sheet-like structure is observed, although some distinct pores are retained. The corresponding SEM images of the dissolved fibers can be found in the supplemental information. 


\subsubsection{High molecular weight heparin, poly(ethylene glycol) and polyethylene oxide}

Control experiments are also performed with a blend of polymers with the identical composition as the cross-linked, equilibrated hydrogel. The composition of the uncross-linked polymers and carrier polymer is $1 \mathrm{wt} \%$ HMWH: 1 wt $\%$ PEG $\left(M_{n}=5000 \mathrm{~g} / \mathrm{mol}\right)$ : 2 wt $\%$ PEO. All samples are observed to spin continuously for 3 hours, resulting in smooth fibers at 14.5 and $16.7 \mathrm{~cm}$ plate separations and fibers sparsely populated with elliptical beads for the $15.5 \mathrm{~cm}$ plate separation. The fibers are shown in Figure 1a. After dissolving the PEO, the microstructure partially dissolves and only very thin fibers remains, Figure $1 b$.

\subsection{Hydrogel electrospinning}

The first hydrogel spinning experiments study the time a hydrogel is able to spin during the gelation reaction and the associated rheological properties. Second, batch experiments are performed that test the spinning of an equilibrated, cross-linked hydrogel with rheological properties chosen from the results of the reacting gel. Note that the concentrations and functionalities of the materials selected for equilibrated and kinetically evolving electrospinning are different. This is necessary, as the aim of kinetically evolving electrospinning is to correlate the change in spinnability with the material's rheological properties as the material transitions from a sol to a gel. From these results, concentrations of the equilibrated solutions are selected for their spinnability even after the materials have gelled into a viscoelastic solid with a low cross-link density. All control experiment microstructures after dissolution significantly differ from the cross-linked hydrogels in the following.

\subsubsection{A reacting solution of high molecular weight heparin $(f=3.9)$, poly(ethylene glycol) $\left(M_{n}=2000 \mathrm{~g} / \mathrm{mol}, f=2\right)$ and polyethylene oxide \\ A solution of $4 \mathrm{wt} \%$ HMWH $(f=3.9)$ : $1 \mathrm{wt} \%$ PEG $\left(M_{n}=2000 \mathrm{~g} / \mathrm{mol}\right.$, $f=2)$ : $1 \mathrm{wt} \%$ PEO is spun at a height of $15.5 \mathrm{~cm}$, an applied voltage of $25 \mathrm{kV}$ and a volumetric flow rate of $750 \mu \mathrm{L} / \mathrm{hr}$ during the gelation reaction. This hydrogel composition is chosen because it gels over a time period of 45 minutes. The long gel time enables the solution to be loaded into the electro- spinning apparatus and spun throughout the sol-gel transition. Immediately after initiation of the gelation reaction, the hydrogel has a shear viscosity of $\eta \approx 4 \mathrm{mPa} \cdot \mathrm{s}$. This solution is not viscous enough to spin. As the viscosity}



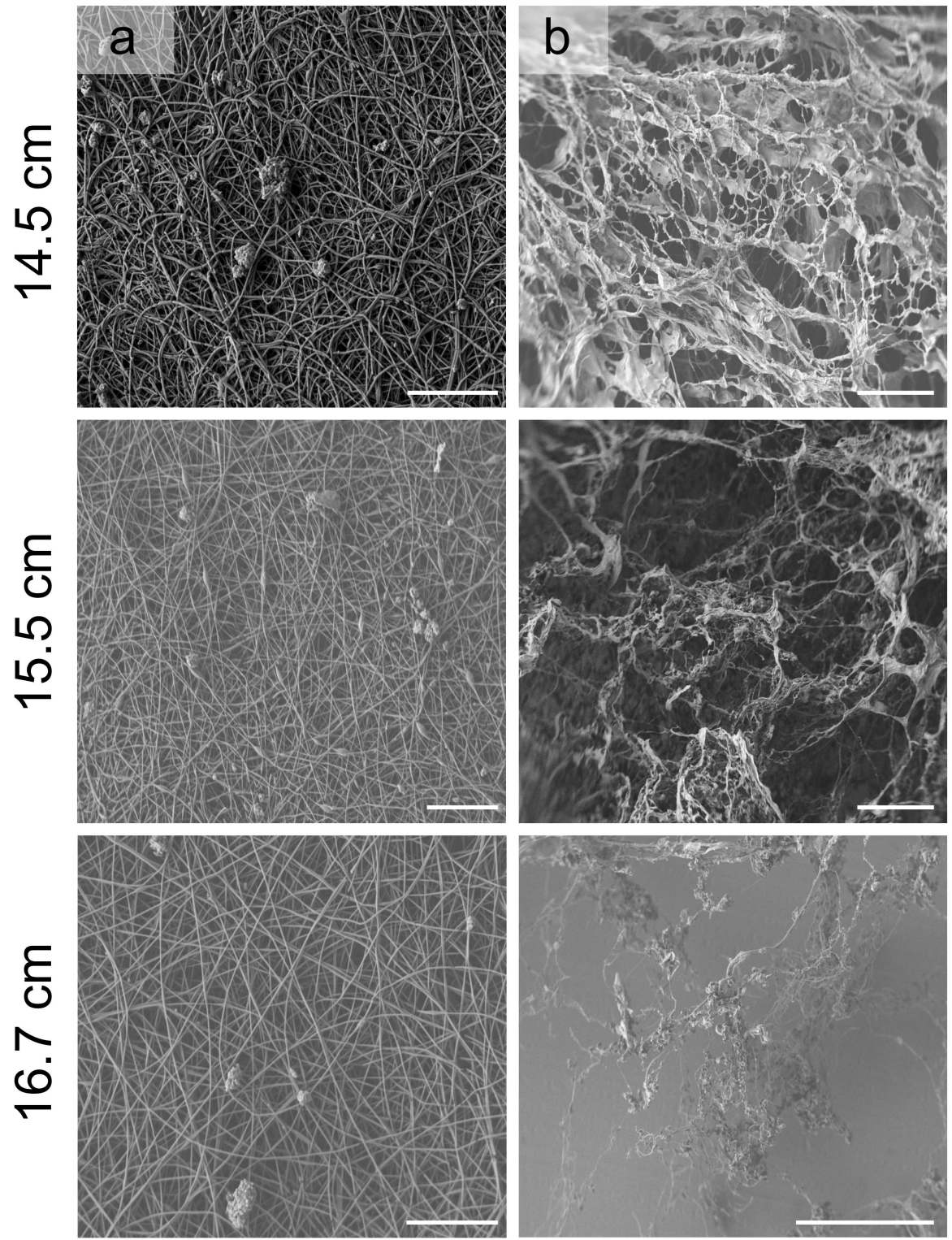

Figure 1: SEM images of (a) fiber mats and (b) dissolved fibers for $1 \mathrm{wt} \%$ HMWH: 1 wt\% PEG $\left(M_{n}=5000 \mathrm{~g} / \mathrm{mol}\right): 2 \mathrm{wt} \%$ PEO from top to bottom for plate separations of 14.5 , 15.5 and $16.7 \mathrm{~cm}$, scalebars on left images are $10 \mu \mathrm{m}$ and on right images are $100 \mu \mathrm{m}$. 
increases, the solution starts to spin. This occurs at 18 minutes when the solution viscosity reaches $8.5 \mathrm{mPa} \cdot \mathrm{s}$, and continues for 3 minutes when the viscosity is $14 \mathrm{mPa} \cdot \mathrm{s}$. The spinning ends well before the critical gelation time of 45 minutes. This range of spinning conditions is illustrated by the solid black symbols in Figure 2a, which shows multiple particle tracking microrheology measurements of the hydrogelation reaction. A brightfield image of the jet spinning is shown in Figure $2 \mathrm{~b}$ and a SEM of the resulting fibers with a bed-on-string morphology are shown in Figure 2c. As the network continues to develop the solution becomes too viscous and eventually gels. The material develops a sufficiently high modulus that leads to extrusion out of the apparatus.

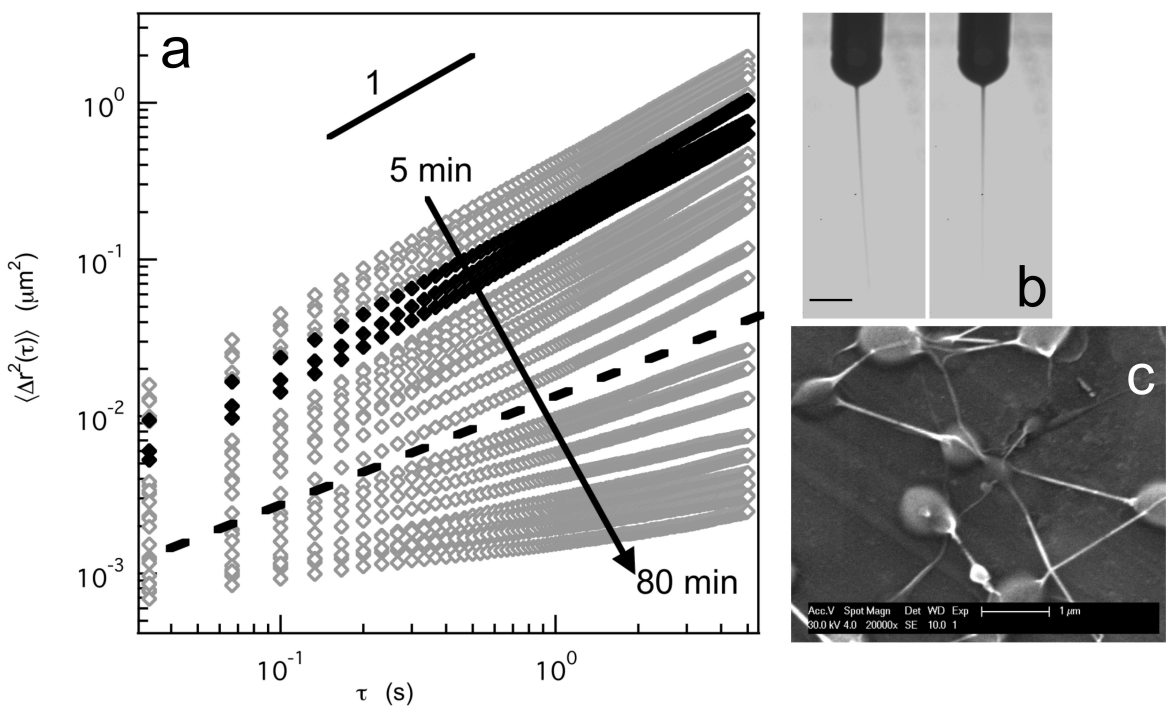

Figure 2: Electrospinning of $4 \mathrm{wt} \%$ HMWH $(f=3.9): 1 \mathrm{wt} \%$ PEG $\left(M_{n}=2000 \mathrm{~g} / \mathrm{mol}\right.$, $f=2$ ): 1 wt\% PEO. (a)MPT measurements of the gelation reaction, the solid black markers indicate the time periods when continuous electrospinning is observed and the dashed line indicates the sol-gel transition. (b) A brightfield image of the fluid jet, scalebar is $0.25 \mathrm{~mm}$, and (c) an SEM image of the fibers.

\subsubsection{Equilibrated solution of high molecular weight heparin $(f=6.3)$, poly(ethylene} glycol) $\left(M_{n}=5000 \mathrm{~g} / \mathrm{mol}, f=2\right)$ and polyethylene oxide

The concentration for the equilibrated hydrogel solution is $1 \mathrm{wt} \% \mathrm{HMWH}$ $(f=6.3): 1 \mathrm{wt} \%$ PEG $\left(M_{n}=5000 \mathrm{~g} / \mathrm{mol}, f=2\right): 2 \mathrm{wt} \%$ PEO. This hydrogel is a weak viscoelastic solid after equilibration. The control experiment 
concentration (discussed above) was selected to match the conditions used here. To electrospin the cross-linked hydrogel, the same parameters as the control experiments are used, $25 \mathrm{kV}, 750 \mu \mathrm{L} / \mathrm{hr}$ and plate separation distances of $14.5,15.5$ and $16.7 \mathrm{~cm}$. Electrospinning is intermittent except for the hydrogel spun at a separation distance of $15.5 \mathrm{~cm}$.

The fiber diameter decreases as the separation distance increases. This corresponds to a diminishing number of elliptical beads, shown in Figure 3a. After dissolving out the PEO, a porous microstructured material is recovered for all separations. The corresponding SEM images are shown in Figure 3b.

\section{Discussion}

The aim of this work is to demonstrate the utility of a rheological material characterization prior to processing a hydrogel with electrospinning. Using MPT measurements of the hydrogelation reaction and a previously developed gelation state diagram, the composition of the hydrogel is chosen a priori to assure spinnability and adjust the desired microstructure. This eliminates the trial and error search to find the appropriate material properties thereby conserving both material and time [45, 46, 47].

Fibers formed during the electrospinning process can be subject to many types of instabilities that change the fiber morphology or inhibit fluid jet from forming in the first place. In our experiments, four types of behavior are observed as the charged fluid leaves the needle and moves towards the ground plate. The first is continuous fiber spinning. The fiber begins spinning once the voltage is applied and spins without interruption for hours until either no sample remains or the process is halted. The next type is intermittent spinning. During this process, a fiber will spin continuously for a period of a few seconds to a few minutes and will then stop. After the spinning stops, a new droplet forms at the tip of the needle that detaches upon reaching a critical size to form a new Taylor cone and continue spinning. Electrospraying, which is characterize by the disintegration of the fluid jet into droplets, is also observed when the electric field is too large. Spraying is used in technological applications such as ink jet printing, but is obviously undesirable in electrospinning [57]. The final behavior is extrusion. This is specific for the electrospinning of a gelling system, and occurs once a gelation reaction has advanced to the point in which the flow behavior of the solution is dominated by the developing elasticity and yield stress. Here, no fibers 

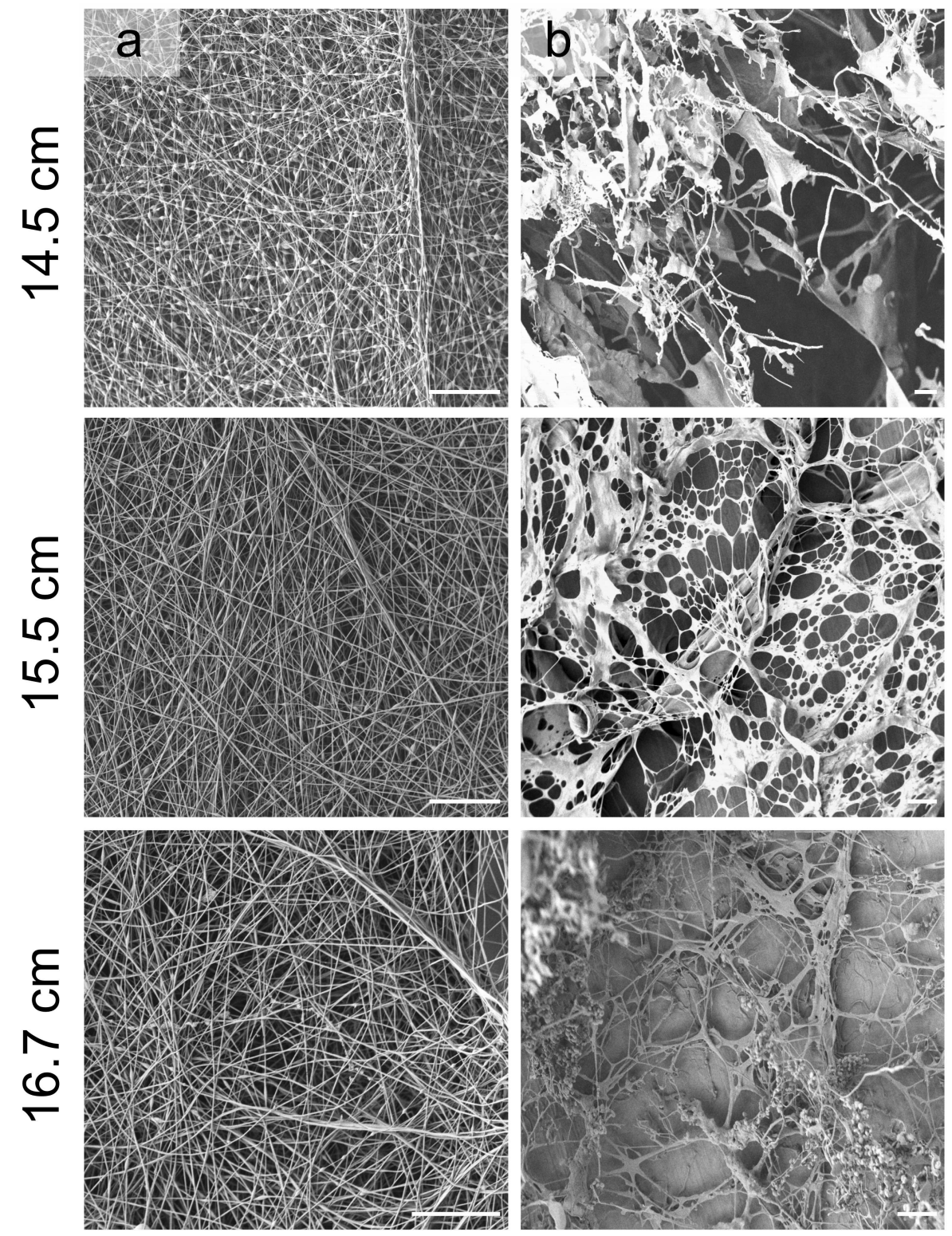

Figure 3: Electrospun structures obtained with the equilibrated cross-linked hydrogel. SEM images of (a) fiber mats and (b) dissolved fibers for $1 \mathrm{wt} \% \mathrm{HMWH}(f=6.3): 1 \mathrm{wt} \%$ PEG $\left(M_{n}=5000 \mathrm{~g} / \mathrm{mol}, f=2\right): 2 \mathrm{wt} \%$ PEO from top to bottom for plate separations of $14.5,15.5$ and $16.7 \mathrm{~cm}$, all scalebars are $10 \mu \mathrm{m}$. 
form, and the material cannot deform viscously, causing it to simply extrude out of the needle.

The resulting fiber morphology from electrospinning is also important. Generally, fibers have diameters on the order of a few hundred nanometers to a few micrometers. For the fibers spun in this investigation, three different fiber morphologies are observed: smooth fibers, fibers with spherical beads and fibers with elliptical beads. The formation of a bead-on-string structure depends on the transient extensional viscosity of the polymer solution jet $[58,59,56,60,61]$. For the case of electrospinning, the bead formation is due to axisymmetric capillary instabilities in the fluid thinning region of the jet $[2,62,63,64]$. Reneker states that the solvent viscosity, surface tension and net charge density play a role in bead formation. Also, the interfacial rheological properties may cause the development of capillary instabilities $[63,64,36]$. The role of elasticity on the extensional viscosity, and therefore the electrospinnability and bead formation has been studied in detail by Rutledge et al., with a focus on solutions with low polymer concentrations $[64,65]$. Lee et al. study the formation of beads in polystyrene fibers. They observe that not only can bead formation be explained by the electrospinning parameters, but the bead morphology can be controlled and changed. Beads can change from circular to elongated shapes by increasing the polymer concentration, plate separation distance or applied voltage. Therefore, engineering the electrospinning process and polymer solution properties can eliminate or enhance bead formation on fibers.

In order to control the fiber formation and diminish the development of bead-on-string structures, all polymer solutions in this study are spun with $\mathrm{PEO}$ as a carrier polymer. The optimal carrier polymer concentration to achieve fibers for the hydrogel spinning was determined for the selected hydrogel compositions to be $2 \mathrm{wt} \% \mathrm{PEO}$, as described in the supplementary information. To determine the effect of cross-linking, we compare the microstructure from the cross-linked hydrogel samples with the uncross-linked control samples of the same composition after dissolution of the PEO carrier polymer. Three different microstructures are observed after the PEO is dissolved. First, a sheet-like structure results from uncross-linked fibers that have dissolved but were not fully washed away during the gentle dissolution procedure. The remaining polymer precipitates upon lyophilization and fuses the non-dissolved fibers together. The next microstructure is a partially dissolved network. The remaining fibers are very thin and the structure lacks the rigidity needed for a mimic of a cell scaffold. The third observed struc- 
ture is a porous, well-defined microstructure as shown in Figure $3 \mathrm{~b}, 15.5 \mathrm{~cm}$. This structure is only observed in the cross-linked hydrogel networks and will be discussed in detail later in this section.

\subsection{Control experiments}

Control experiments performed with PEO solutions of $1-3 \mathrm{wt} \%$ did not result in a total dissolution of the electrospun fibers. However, the remaining PEO fails to form a scaffold structure, Detailed results are provided in the supplementary information.

The control experiments with a blend of non-functionalized, uncrosslinked hydrogel components 1 wt\% HMWH $(f=0)$ : 1 wt\% PEG $\left(M_{n} 5\right.$ 000, $f=0): 2$ wt $\%$ PEO form fibers with diameters that vary with the point-plate separation distance. The smallest diameter measured, $0.28 \mu \mathrm{m}$ at a separation of $15.5 \mathrm{~cm}$, along with the formation of beads. Dissolution of the carrier polymer also results in the dissolution of the non-functionalized polymers, leaving only thin fibers. The resulting pores in this thin-fibered structure are large, $13-24 \mu \mathrm{m}$, and would not be appropriate as a scaffold for biological applications. The fiber diameters and pore sizes of the control experiments are listed in Table 2. The remaining fiber structure of the hydrogels after dissolution is caused purely by physical entanglements that are not sufficient to create a structured scaffold.

\subsection{Hydrogel electrospinning}

The effects of gelation reaction on the spinning process is studied using a 4 wt $\%$ HMWH $(f=3.9)$ : 1 wt $\%$ PEG $\left(M_{n}=2000 \mathrm{~g} / \mathrm{mol}, f=2\right)$ : 1 wt\% PEO solution the solution does not initially spin. As the gelation reaction proceeds, spinning eventually occurs over a period of $5-8$ minutes after initiating the gelation reaction (see Figure 2). Within this period, the material has polymer clusters forming, which increases the solution viscosity and elasticity, but is still prior to the liquid-solid transition that occurs at the gel point. Finally, as the hydrogel continues to evolve into a stiff gel, the material extrudes from the needle instead of spinning. This experiment demonstrates that an equilibrated gel with a high modulus will not spin, but that there is a window of lower cross-linking density over which the electrospinning of hydrogels is possible. Outgoing from this, we are able to use the gelation state diagram obtained from microrheology to identify hydrogel compositions that are favorable for continuous spinning. 
Gelation state diagrams are created from MPT characterization of equilibrated hydrogels. As shown in Figure 4, the logarithmic slope of the mean-squared displacement is plotted as a function of the backbone polymer concentration (heparin) and the cross-linker concentration (PEG). State diagrams are useful for quickly determining the gel lines as a function of hydrogel composition $[45,46,66,67,47]$. Each box in the diagram represents an equilibrated hydrogel. The logarithmic slope of the mean-squared displacement, $\alpha=\frac{\mathrm{d} \log \left\langle\Delta r^{2}(\tau)\right\rangle}{\mathrm{d} \log \tau}$, indicates the state of the material that the probes are embedded in, as shown in Figure 2. A unity (logarithmic) slope $\alpha=1$ at long delay times $\tau$ indicates that the probe particles are freely diffusing in a Newtownian fluid. A slope that is less than one but greater than zero is indicative of a viscoelastic fluid or solid. In the viscoelastic regime, the critical relaxation exponent $n$ distinguishes the liquid from the solid: for a fluid, $\alpha>n$, while a viscoelastic solid has $\alpha<n$. The critical exponent depends on the material of interest $[68,69,70,71]$, but has a value between 0.4 and 0.6 for PEG-HMWH hydrogels [46, 67, 47, 72]. Finally, $\alpha \approx 0$ indicates that the probe particles appear arrested in an elastic solid.

The color of each box in Figure 4 is the logarithmic slope of the MSD. Red indicates a stiff gel and pale blue indicate a liquid. For example, a composition of $3 \mathrm{wt} \%$ HMWH $(f=3.9): 2 \mathrm{wt} \%$ PEG $\left(M_{n}=10000 \mathrm{~g} / \mathrm{mol}, f=2\right)$, is a strong gel at equilibrium, and therefore is predicted not to electrospin, as indicated on the gelation state diagram, Figure 4a. An attempt to spin this gel is shown in Figure 4b, and clearly only extrusion of the gelled material is achieved. Eventually, the extruded gel becomes subject to a whipping instability as the length of the fibers increases and results in breakage.

We now discuss our results for spinning an equilibrated hydrogel. In this case, the gelation state diagram, Figure 5, is used to select a gel composition for which the material rheology is sufficiently viscous for electrospinning, but the cross-linking density at equilibrium does not suppress the formation of a thinning jet. First, consider the conditions required to form a Taylor cone in a weak viscoelastic solid. The material must have a maximum yield stress, $\tau_{y, \max }$, set by the electric stress, $\tau_{y, \max } \approx \epsilon_{0} E^{2}$. At a typical field strength used to spin the hydrogel in this work, $E=1.6 \mathrm{kV} / \mathrm{cm}$, the maximum yield stress is approximately $\tau_{y, \max } \approx 0.25 \mathrm{~Pa}$. Using the equilibrium elastic modulus, $G_{e}$, and critical strain, $\gamma_{c}$, the yield stress is estimated as $\tau_{y} \sim G_{e} \gamma_{c}$. The critical strain for similar hydrogels is $\gamma_{c} \sim 10 \%$ [73]. Therefore, the equilibrium modulus of the material must be less than $2.5 \mathrm{mPa}$. 

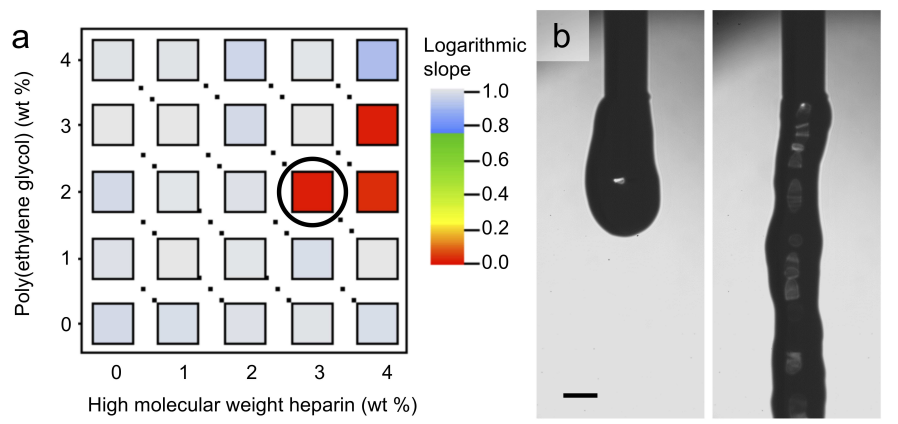

Figure 4: Electrospinning of a stiff hydrogel. (a) The gelation state diagram for HMWH $(f=3.9)$ and PEG $\left(M_{n}=10000 \mathrm{~g} / \mathrm{mol}\right)$ hydrogel. (b) A brightfield image of $3 \mathrm{wt} \%$ HMWH $(f=3.9): 2 \mathrm{wt} \%$ PEG $\left(M_{n}=10000 \mathrm{~g} / \mathrm{mol}\right)$ hydrogel extruding from needle, scalebar $0.25 \mathrm{~mm}$. (a) Reprinted with permission from K.M. Schultz, A. D. Baldwin, K. L. Kiick and E. M. Furst, Macromolecules, 2009, 42, 5310-5316. Copyright 2009 American Chemical Society.

From Figure 5, a weak gel with $G_{e}<2.5 \mathrm{~Pa}$ is expected at the concentration of $1 \mathrm{wt} \% \mathrm{HMWH}(f=6.3): 1 \mathrm{wt} \% \mathrm{PEG}\left(M_{n}=5000 \mathrm{~g} / \mathrm{mol}, f=2\right)$. This composition is close to the gel line boundaries; the mean-squared displacement logarithmic slope is $\alpha=0.2$, and the critical relaxation exponent is $n=0.48$. With the addition of $2 \mathrm{wt} \% \mathrm{PEO}$, this equilibrated hydrogel spins for hours continuously into fibers at the plate-needle separation 15.5 $\mathrm{cm}$ (see Figure 2) and spins intermittently at other plate separations. During the subsequent $\mathrm{PEO}$ dissolution, a porous fiber structure is retained and the fibers further cross-link to create a unique network. The lightly crosslinked gel can re-form due to the number of unreacted cross-linking sites. Microstructures are formed in quasi-two dimensions, therefore, between the small porous sheets there are large pores or holes in the $z$ direction. This leads to a large standard deviation in the pore size, as summarized in Table 2. The typical mesh obtained from electrospinning and subsequent dissolution of the carrier polymer exhibits a distinct microstructure of the hydrogel (see Figure 3) that is not seen in the control experiments with the uncross-linked components.

\subsection{Comparison of uncross-linked and cross-linked fibers}

In Table 2 the results of spinning an equilibrated cross-linked hydrogel are compared to the spinning of uncross-linked polymer mixtures with the same 


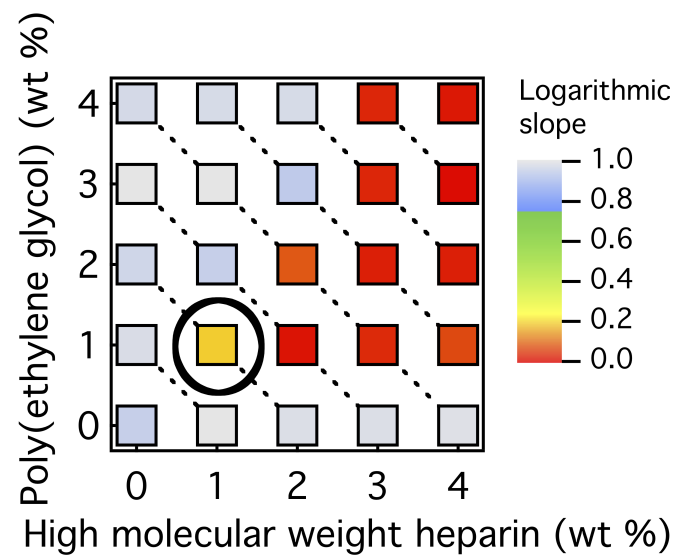

Figure 5: Gelation state diagram for HMWH $(f=6.3)$ : PEG $\left(M_{n}=5000 \mathrm{~g} / \mathrm{mol}\right)$ with the electrospun composition highlighted. Reprinted with permission from K.M. Schultz, A. D. Baldwin, K. L. Kiick and E. M. Furst, Macromolecules, 2009, 42, 5310-5316. Copyright 2009 American Chemical Society.

concentrations of heparin, PEG and PEO. Control experiments, described in detail above and in the supplemental materials, are performed on all of the combinations of polymer concentrations (with reactive and unreactive polymers) to ensure that the results achieved for the reactive solution are unique to that material.

Continuous spinning for both the uncross-linked and cross-linked solutions requires the addition of $\mathrm{PEO}$. This is not substantially influenced by changes in the weak degree of cross-linking in pre-gel states or weak, equilibrated hydrogels. The cross-linking, however, has a notable influence on the fiber morphology. The cross-linked hydrogel fibers have fewer beads than the uncross-linked control solutions due to an increase of the viscoelastic properties of the material.

The hydrogel materials experience significant stress during spinning. We expect the spinning to disrupt the polymer network, but understanding the full extent of network degradation is beyond current capabilities. However, one expects large portions of the network will remain intact as large polymer clusters. Furthermore, the number of unreacted cross-links (estimated from the modulus in earlier papers) enables the hydrogel to reform after spinning, at least in part. The effect of this cross-linking is clearly seen when comparing the uncross-linked control samples after dissolution of the PEO carrier. 
Table 2: Viscosity, $\eta$, fiber diameter, $d_{f i b e r}$, and pore size, $l_{\text {pore }}$, for all spun solutions.

\begin{tabular}{|c|c|c|c|c|}
\hline $\begin{array}{c}\text { Solution } \\
\text { concentration }\end{array}$ & height (cm) & $\eta(\mathrm{mPa} \cdot \mathrm{s})$ & $d_{\text {fiber }}(\mu \mathrm{m})$ & $l_{\text {pore }}(\mu \mathrm{m})$ \\
\hline $3 \% \mathrm{PEO}$ & 14.5 & 200 & $0.42 \pm 0.35$ & $29 \pm 63$ \\
\hline $3 \% \mathrm{PEO}$ & 15.5 & 200 & $0.31 \pm 0.11$ & $12 \pm 8.3$ \\
\hline $3 \% \mathrm{PEO}$ & 16.7 & 200 & $0.28 \pm 0.10$ & - \\
\hline $\begin{array}{c}\text { 1\% HMWH: } 1 \% \\
\text { PEG: }\end{array}$ & & & & \\
\hline $2 \% \mathrm{PEO}$ & 14.5 & 200 & $0.30 \pm 0.11$ & $24 \pm 15$ \\
\hline $\begin{array}{c}\text { 1\% HMWH: } 1 \% \\
\text { PEG: }\end{array}$ & & & & \\
\hline $2 \% \mathrm{PEO}$ & 15.5 & 200 & $0.28 \pm 0.11$ & $16 \pm 16$ \\
\hline $\begin{array}{c}\text { 1\% HMWH: } 1 \% \\
\text { PEG: }\end{array}$ & & & & \\
\hline $2 \% \mathrm{PEO}$ & 16.7 & 200 & $0.39 \pm 0.13$ & $13 \pm 21$ \\
\hline $\begin{array}{c}1 \% \text { HMWH } \\
(f=6.3): \\
1 \% \text { PEG }(f=2): \\
2 \% \text { PEO }\end{array}$ & 14.5 & - & $0.20 \pm 0.09$ & $12 \pm 12$ \\
\hline $\begin{array}{c}1 \% \text { HMWH } \\
(f=6.3): \\
1 \% \text { PEG }(f=2): \\
2 \% \text { PEO }\end{array}$ & 15.5 & - & $0.28 \pm 0.09$ & $8.5 \pm 9.1$ \\
\hline $\begin{array}{c}1 \% \text { HMWH } \\
(f=6.3): \\
1 \% \text { PEG }(f=2): \\
2 \% \text { PEO }\end{array}$ & 16.7 & - & $0.36 \pm 0.19$ & $8.0 \pm 14$ \\
\hline
\end{tabular}

Upon dissolution of the carrier polymer, only the cross-linking samples retain a distinct microstructure. The resulting porous structure of both cross-linked and uncross-linked solutions of 1 wt\% HMWH: 1 wt\% PEG: 2 wt\% PEO are shown for comparison in Figure 6. Figure 6a shows the uncross-linked microstructure of the unfunctionalized polymer mixture while Figure $6 \mathrm{~b}$ shows the microstructure of the cross-linked hydrogel material. The cross-linked hydrogel material microstructure fuses together further upon dissolution, but 


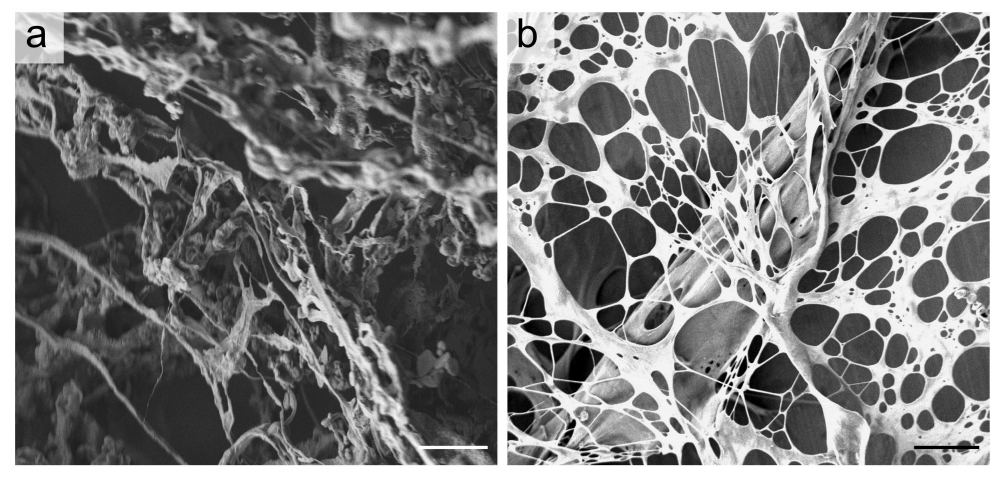

Figure 6: SEM images of dissolved fibers for (a) $1 \mathrm{wt} \%$ HMWH $(f=0): 1 \mathrm{wt} \%$ PEG $\left(M_{n}\right.$ $5000, f=0): 2 \mathrm{wt} \%$ PEO and (b) $1 \mathrm{wt} \%$ HMWH $(f=6.3): 1 \mathrm{wt} \%$ PEG $\left(M_{n} 5000, f=2\right)$ : $2 \mathrm{wt} \%$ PEO for $15.5 \mathrm{~cm}$ plate separation, scalebars are $10 \mu \mathrm{m}$.

retains a porous structure, while the porous structure of the uncross-linked material dissolves during the removal of PEO. There is also a noticeable difference in the pore size with the cross-linked material yielding pores on the length scale of a cell. The addition of PEO to a hydrogel scaffold that is not electrospun would cause an increase in the pore size of the hydrogel scaffold, but we would not expect an order-of-magnitude increase that we achieve by processing the material with electrospinning.

\section{Conclusions}

The focus of this work is the processing of PEG-HMWH hydrogel material using electrospinning. To electrospin a hydrogel we must first identify the material properties that are suitable for this process. The extensional viscosity of the (gelling) hydrogel material must be such that it can flow and form stable filaments during spinning, but is not too stiff or viscous that the material only extrudes from the charging needle. In previous work, the creation of gelation state diagrams using high-throughput microrheology is described and refined [45, 46, 66, 67, 47]. Within the current study it was shown that these gelation state diagrams allow us to predict the composition of the hydrogel with the required viscoelastic properties to facilitate electrospinning, eliminating unnecessary time and material wasted on trial and error experiments. For the present study, a shear viscosity of $\eta \approx 8.5-14 \mathrm{mPa} \cdot \mathrm{s}$ (and the related extensional and non-linear viscosities) have shown to be optimal for 
electrospinning experiments. Not only does this work emphasize the application of the state diagram, but it also illustrates the processing of this material into a porous network for therapeutic and biological applications, such as tissue regeneration or wound healing. Using electrospinning, a porous network of fibers is created with diameters ranging from a few hundred nanometers to micrometers and pore sizes on the order of tens of micrometers, a length scale similar to the ECM. The fibers fabricated of PEG-HMWH hydrogel have the physically relevant length scales to mimic the extracellular matrix. This recapitulates biophysical cues necessary for applications such as tissue regeneration, would healing and stem cell culture.

\section{Acknowledgements}

Funding for this work was provided by the National Science Foundation (grant nos. CBET-0730292 for microrheology and DGE-0221651 for synthesis), the European research council (ERC-2007-StG starting grant 203043 NANOFIB for the electrospinning) and the National Institutes of Health (5P20-RR016472-10). E.M.F. acknowledges financial support for biomaterial microrheology under P20-RR017716. The contents of the manuscript are the sole responsibility of the authors and do not necessarily reflect the official views of the National Institutes of Health nor of the National Center

for Research Resources. K.M.S. acknowledges financial support by the NSF Graduate Research Fellowship Program.

\section{References}

[1] J. Zeleny, Phys. Rev. 3 (1914) 69-91.

[2] M. E. Helgeson, K. N. Grammatikos, J. M. Dietzel, N. J. Wagner, Polymer 49 (2008) 2924-2936.

[3] G. C. Rutledge, S. V. Fridrikh, Adv. Drug Delivery Rev. 59 (2007) 1384-1391.

[4] D. H. Reneker, A. L. Yarin, Polymer 49 (2008) 2387-2425.

[5] Y. M. Shin, M. M. Hohman, M. P. Brenner, G. C. Rutledge, Appl. Phys. Lett. 78 (2001) 1149-1151. 
[6] D. H. Reneker, Y. Xin, H. Lee, Y. Lin, K. Liu, Z. Zhong, MRS Proceedings (2009).

[7] S. Tripatanasuwan, D. H. Reneker, Polymer 50 (2009) 1835-1837.

[8] C. P. Barnes, S. A. Sell, E. D. Boland, D. G. Simpson, G. L. Bowlin, Adv. Drug Delivery Rev. 59 (2007) 1413-1433.

[9] D. Liang, B. S. Hsiao, B. Chu, Adv. Drug Delivery Rev. 59 (2007) 1392 1412.

[10] J. Xie, X. Li, Y. Xia, Macromol. Rapid Commun. 29 (2008) 1775-1792.

[11] J. J. Stankus, J. Guan, K. Fujimoto, W. R. Wagner, Biomaterials 27 (2006) 735-744.

[12] W. He, T. Yong, W. E. Teo, Z. Ma, S. Ramakrishna, Tissue Eng. 11 (2005) 1574-1588.

[13] S. A. Sell, G. L. Bowlin, J. Mater. Chem. 18 (2008) 260-263.

[14] M. J. Smith, M. J. McClure, S. A. Sell, C. P. Barnes, B. H. Walpoth, D. G. Simpson, G. L. Bowlin, Acta Biomater. 4 (2008) 58-66.

[15] C. K. Hashi, Y. Zhu, G.-Y. Yang, W. L. Young, B. S. Hsiao, K. Wang, B. Chu, S. Li, PNAS 104 (2007) 11915-11920.

[16] S. J. Lee, J. Liu, S. H. Oh, S. Soker, A. Atala, J. J. Yoo, Biomaterials 29 (2008) 2891-2898.

[17] J. P. Stegemann, S. N. Kaszuba, S. L. Rowe, Tissue Eng. 13 (2007) 2601-2613.

[18] M. Stevens, J. H. George, Science 310 (2005) 1135-1138.

[19] S. J. Lee, S. H. Oh, J. Liu, S. Soker, A. Atala, J. J. Yoo, Biomaterials 29 (2008) 1422-1430.

[20] S. J. Lee, J. J. Yoo, G. J. Lim, A. Atala, J. Stitzel, J. Biomed. Mater. Res. A 83A (2007) 999-1008.

[21] B. W. Tillman, S. K. Yazdani, S. J. Lee, R. L. Geary, A. Atala, J. J. Yoo, Biomaterials 30 (2009) 583-588. 
[22] Y. Hong, S.-H. Ye, A. Nieponice, L. Soletti, D. A. Vorp, W. R. Wagner, Biomaterials 30 (2009) 2457-2467.

[23] Y. Dong, T. Yong, S. Liao, C. K. Chan, M. M. Stevens, S. Ramakrishna, Tissue Eng. Part A 16 (2008) 283-298.

[24] C. Gentilini, Y. Dong, J. R. May, S. Goldoni, D. E. Clarke, B.-H. Lee, E. T. Pashuck, M. M. Stevens, Adv. Healthcare Mater. (2012) DOI: 10. 1002/adhm.201200036.

[25] E. S. Place, J. H. George, C. K. Williams, M. M. Stevens, Chem. Soc. Rev. 38 (2009) 1139-1151.

[26] N. W. Garrigues, D. Little, O. C. J., F. Guilak, J. Mater. Chem. 20 (2010) 8962-8968.

[27] Y. Dong, S. Liao, M. Ngiam, C. K. Chan, S. Ramakrishna, Tissue Eng. Part B 15 (2009) 333-351.

[28] J. Y. Lee, C. A. Bashur, A. S. Goldstein, C. A. Schmidt, Biomaterials 30 (2009) 4325-4335.

[29] J. Y. Lee, C. A. Bashur, N. Gomez, A. S. Goldstein, C. E. Schmidt, J. Biomed. Mater. Res. A 92A (2010) 1398-1406.

[30] C. A. Bashur, C. A. Milroy, L. Forciniti, A. S. Goldstein, C. E. Schmidt, IEEE Trans. Nanobiosci. 11 (2012) 15-21.

[31] S. A. Zawko, C. E. Schmidt, J. Biomed. Mater. Res. A 98A (2011) 287295.

[32] W.-J. Li, H. Chiang, T.-F. Kuo, H.-S. Lee, C.-C. Jiang, R. S. Tuan, J. Tissue Eng. Regen. Med. 3 (2009) 1-10.

[33] F. H. Chen, K. T. Rousche, R. S. Tuan, Nat. Clin. Pract. Rheumatol. 2 (2006) 373-382.

[34] D. Baksh, L. Song, R. S. Tuan, J. Cell Mol. Med. 8 (2004) 301-316.

[35] N. Bhardwaj, S. C. Kundu, Biotechnol. Adv. 28 (2010) 325-347.

[36] O. Regev, S. Vandebril, E. Zussman, C. Clasen, Polymer 51 (2010) 2611-2620. 
[37] S. I. Stupp, Nano Lett. 10 (2010) 4783-4786.

[38] W. W. Tsai, I. D. Tevis, A. S. Tayi, H. Cui, S. I. Stupp, J. Phys. Chem. B 114 (2010) 14778-14786.

[39] Y. Liu, J. Chen, V. Misoska, G. G. Wallace, React. Funct. Polym. 67 (2007) 461-467.

[40] J. Xie, M. R. MacEwan, A. G. Schwartz, Y. Xia, Nanoscale 2 (2010) 35-44.

[41] Y. Ji, K. Ghosh, B. Li, J. C. Sokolov, R. A. F. Clark, M. H. Rafailovich, Macromol. Biosci. 6 (2006) 811-817.

[42] Y. Ji, K. Ghosh, X. Zheng Shu, B. Li, J. C. Sokolov, G. D. Prestwich, R. A. F. Clark, M. H. Rafailovich, Biomaterials 27 (2006) 3782-3792.

[43] S. H. Kim, S. Kim, S. Nair, E. Moore, Macromolecules 38 (2005) 3719 3723.

[44] Y. Jin, D. Yang, Y. Zhou, G. Ma, J. Nie, J. Appl. Polym. Sci. 109 (2008) 3337-3343.

[45] K. M. Schultz, A. D. Baldwin, K. L. Kiick, E. M. Furst, Soft Matter 5 (2009) 740-742.

[46] K. M. Schultz, A. D. Baldwin, K. L. Kiick, E. M. Furst, Macromolecules 42 (2009) 5310-5316.

[47] K. M. Schultz, E. M. Furst, Soft Matter (2012) DOI: $10.1039 / \mathrm{c} 2 \mathrm{sm} 25187 \mathrm{f}$.

[48] S. Liu, R. Maheshwari, K. L. Kiick, Macromolecules 42 (2009) 3-13.

[49] T. Nie, A. Baldwin, N. Yamaguchi, K. L. Kiick, J. Controlled Release 122 (2007) 287-296.

[50] T. Savin, P. S. Doyle, Biophys. J. 88 (2005) 623-638.

[51] V. Breedveld, D. J. Pine, J. Mater. Sci. 38 (2003) 4461-4470.

[52] T. G. Mason, D. A. Weitz, Phys. Rev. Lett. 74 (1995) 1250-1253. 
[53] J. C. Crocker, D. G. Grier, J. Colloid Interface Sci. 179 (1996) 298-310.

[54] J. D. Ferry, Viscoelastic Properties of Polymers, John Wiley and Sons, Inc., third edition, 1980.

[55] R. G. Larson, The Structure and Rheology of Complex Fluids, Oxford University Press, 1999.

[56] L. Campo-Deano, C. Clasen, J. Non-Newtonian Fluid Mech. 165 (2010) 1688-1699.

[57] J. Zeleny, Phys. Rev. 10 (1917) 1-6.

[58] C. Clasen, C. J. Eggers, M. A. Fontelos, J. Li, G. H. McKinley, J. Fluid Mech. 556 (2006) 283-308.

[59] M. S. N. Oliveira, R. Yeh, G. H. McKinley, J. Non-Newtonian Fluid Mech. 137 (2006) 137-148.

[60] C. Clasen, Korea-Aust. Rheol. J. 22 (2010) 331-338.

[61] C. Clasen, P. M. Phillips, L. J. Palangetic, J. Vermant, AIChE J. (2011) DOI: 10.1002/aic.13704.

[62] M. M. Hohman, M. Shin, G. Rutledge, M. P. Brenner, Phys. Fluids 13 (2001) 2221.

[63] H. Fong, I. Chun, D. H. Reneker, Polymer 40 (1999) 4585-4592.

[64] K. H. Lee, H. Y. Kim, H. J. Bang, Y. H. Jung, S. G. Lee, Polymer 44 (2003) 4029-4034.

[65] J. H. Yu, S. V. Fridrikh, G. C. Rutledge, Polymer 47 (2006) 4789-4797.

[66] K. M. Schultz, E. M. Furst, Lab Chip 11 (2011) 3802-3809.

[67] K. M. Schultz, A. V. Bayles, A. D. Baldwin, K. L. Kiick, E. M. Furst, Biomacromolecules 12 (2011) 4178-4182.

[68] D. Adolf, J. E. Martin, Macromolecules 23 (1990) 3700-3704.

[69] F. Chambon, H. H. Winter, J. Rheol. 31 (1987) 683-697. 
[70] H. H. Winter, Polym. Eng. Sci. 27 (1987) 1698-1702.

[71] T. H. Larsen, K. M. Schultz, E. M. Furst, Korea-Aust. Rheol. J. 20 (2008) 165-173.

[72] K. M. Schultz, A. D. Baldwin, K. L. Kiick, E. M. Furst, Macro Letters (2012) DOI: 10.1021/mz300106y.

[73] N. Yamaguchi, B.-S. Chae, L. Zhang, K. L. Kiick, E. M. Furst, Biomacromolecules 6 (2005) 1931-1940. 